\title{
Nonlinear Aeroelastic Trim of Very Flexible Aircraft Described by Detailed Models
}

\author{
Cristina Riso* \\ University of Michigan, Ann Arbor, Michigan 48109 \\ Fausto G. Di Vincenzo \\ MSC.Software Toulouse, 31300 Toulouse, France \\ Markus Ritter \\ DLR, German Aerospace Center, 37073 Göttingen, Germany \\ Carlos E. S. Cesnik $\underline{\underline{s}}$ \\ University of Michigan, Ann Arbor, Michigan 48109 \\ and \\ Franco Mastroddi I \\ University of Rome “La Sapienza,” Rome 00184, Italy \\ DOI: $10.2514 / 1 . C 034787$
}

\begin{abstract}
This Paper presents an efficient algorithm for the nonlinear aeroelastic trim analysis of very flexible aircraft described by detailed models. The algorithm is based on a novel inertia relief technique for large displacements and is applicable to fluid-structure iteration frameworks coupling generic structural and aerodynamic solvers, including high-fidelity commercial solvers. The methodology is tested on a low-order model of the University of Michigan's X-HALE experimental vehicle in order to compare to reference results for a typical steady rectilinear flight condition. Nonlinear aeroelastic trim analyses conducted at different flight speeds show that the proposed approach gives a smooth and fast convergence to the trim solution, which makes it suitable for high-fidelity aeroelastic design of very flexible aircraft.
\end{abstract}

\section{Nomenclature}

$a_{2}^{S}, a_{3}^{S} \quad=\quad$ lateral and vertical acceleration components of stability axes, $\mathrm{m} / \mathrm{s}^{2}$

$D \quad=$ rigid-body mode matrix

$\hat{\boldsymbol{E}}_{q} \quad=$ steady aerodynamic force matrix for nodal degrees of freedom

$\hat{\boldsymbol{E}}_{u} \quad=\quad$ steady aerodynamic force matrix for trim variables

$\hat{\boldsymbol{f}}^{A} \quad=$ aerodynamic nodal load vector

$\hat{\boldsymbol{f}}^{A 0}=$ aerodynamic nodal load vector due to initial incidence, camber, and twist

$\hat{f}^{I} \quad=$ inertia relief nodal load vector

$\hat{\boldsymbol{f}}^{W} \quad=$ gravity nodal load vector

$\hat{\boldsymbol{K}}, \hat{\boldsymbol{K}}^{A}=$ structural and aeroelastic stiffness matrices

$\hat{\boldsymbol{M}} \quad=$ mass matrix

$N_{l}, N_{r}, N_{t}=$ number of leftover, reference (support), and total degrees of freedom

$N_{u} \quad=$ number of trim variables

$n_{a}, n_{s}, n_{t}=$ number of additional, load-step, and total iterations

$p^{S} \quad=$ roll rate in stability axes, $\mathrm{rad} / \mathrm{s}$

$\hat{\boldsymbol{q}}=$ vector of nodal degrees of freedom

$q_{D} \quad=$ freestream dynamic pressure, $\mathrm{N} / \mathrm{m}^{2}$

$q^{S} \quad=$ pitch rate in stability axes, $\mathrm{rad} / \mathrm{s}$

Received 13 October 2017; revision received 4 April 2018; accepted for publication 21 May 2018; published online 13 August 2018. Copyright (C) 2018 by the American Institute of Aeronautics and Astronautics, Inc. All rights reserved. All requests for copying and permission to reprint should be submitted to CCC at www.copyright.com; employ the ISSN 0021-8669 (print) or 1533-3868 (online) to initiate your request. See also AIAA Rights and Permissions www.aiaa.org/randp.

*Research Fellow, Department of Aerospace Engineering, 1320 Beal Avenue.

${ }^{\dagger}$ Presales Engineer Aero, 4 rue du Prof. Pierre Vellas.

${ }^{*}$ Research Engineer, Institute of Aeroelasticity, 10 Bunsenstraße.

${ }^{\S}$ Professor, Department of Aerospace Engineering, 1320 Beal Avenue.

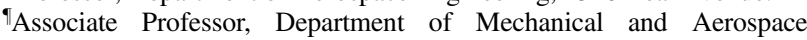
Engineering, via Eudossiana 18.

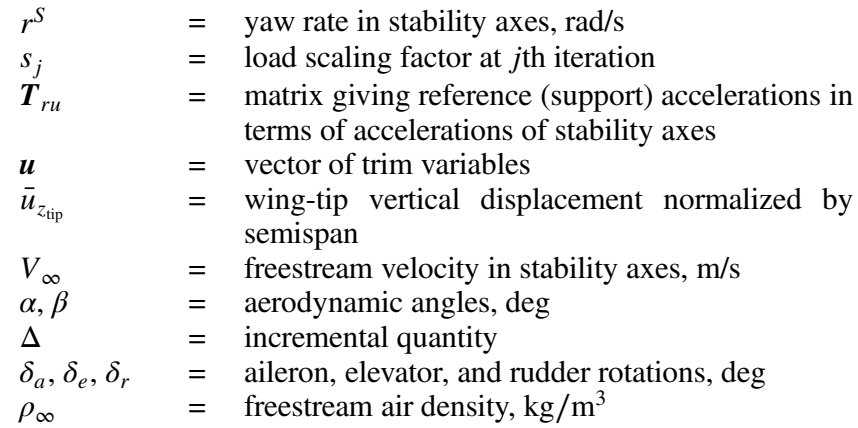

Subscripts
e $\quad=$ trim quantity
$l=$ quantity referring to leftover degree of freedom
$r \quad=\quad$ quantity referring to reference (support) degree of freedom

Superscripts

$\begin{array}{ll}(j) & =\text { quantity evaluated at } j \text { th iteration } \\ \dot{\square}, \ddot{\square} & =\text { first- and second-order time derivatives } \\ \dot{\square} & =\text { nodal quantity }\end{array}$

\section{Introduction}

EXT-GENERATION aircraft will be characterized by very high-aspect-ratio wings and slender lightweight structures, resulting in much larger deflections compared to today's vehicles. Among other consequences of high flexibility, the statically deformed aircraft configuration at trim, which inherently depends on the operating condition, shall be considered as the baseline for aeroelastic design, guidance, and control [1].

The need to account for the effects of large trim deflections when studying very flexible aircraft was pointed out by several authors [1-3] . Linearized dynamic models based on the deformed shape were often found to retain most of the physics of fully nonlinear 
descriptions [1-4]. In contrast, analyses based on the jig shape frequently resulted in poor predictions [1-3] and flight-test failures [5].

At the academic research level, low-order computational tools have been developed to study very flexible aircraft $[2,6]$ modeled as sets of geometrically nonlinear beams $[\underline{7}, 8]$. While such low-order descriptions provide valuable insights into key nonlinear aeroelastic behaviors, design of next-generation vehicles requires high-fidelity modeling to significantly improve performance and reduce late modifications in the design process. Nevertheless, there is still a lack of efficient methodologies to solve nonlinear aeroelastic problems, including trim problems, using detailed structural and aerodynamic models. State-of-the-art commercial solvers used in industrial aeroelastic design [9] allow for using built-up finite element method (FEM) models but neglect structural nonlinearities and assume linear aerodynamics at best corrected with computational fluid dynamic (CFD) data $[9,10]$.

This Paper presents an efficient algorithm for the nonlinear aeroelastic trim analysis of very flexible aircraft described by structural and aerodynamic models of any fidelity, including detailed representations [11]. The algorithm is based on a novel largeamplitude inertia relief technique that generalizes the linear inertia relief analysis implemented in commercial FEM solvers $[12,13]$ in order to simulate the vehicle as unrestrained at each iteration of the nonlinear solution process. To test the proposed methodology, a medium-fidelity computational environment coupling the MSC Nastran nonlinear structural solver SOL 400 [12] with an in-house vortex-lattice method (VLM) code developed at DLR, German Aerospace Center (DLR) [14] is implemented and applied to trim the University of Michigan's X-HALE [15,16]. A low-order description of the aircraft is considered to compare to available results from the University of Michigan's Nonlinear Aeroelastic Simulation Toolbox (UM/NAST) [6] and from a DLR aeroelastic toolbox [14] for a typical steady rectilinear flight condition. Nonlinear aeroelastic trim analyses at different flight speeds show that the proposed approach gives a smooth and fast convergence to the trim solution, which makes it suitable for high-fidelity aeroelastic design of very flexible aircraft.

\section{Aeroelastic Trim Algorithm}

This section describes the proposed algorithm for the nonlinear aeroelastic trim analysis of very flexible aircraft [11]. The methodology is based on a novel large-amplitude inertia relief technique [11] that allows one to simulate an unrestrained vehicle in a nonlinear solution process and is applicable to fluid-structure interaction frameworks coupling generic structural and aerodynamic solvers, including high-fidelity commercial solvers. The role of inertia relief analysis applied to aeroelastic trim problems solved using displacement-based FEM structural formulations is clarified by first recalling the linear aeroelastic trim algorithm implemented in MSC Nastran SOL 144 [9] before introducing the proposed nonlinear approach.

\section{A. Linear Algorithm}

Linear aeroelastic trim analyses conducted using MSC Nastran SOL 144 [9] are based on the equation of motion for a quasi-steady aeroelastic vehicle $[\underline{9}, \underline{17}]$,

$$
\hat{\boldsymbol{M}} \ddot{\hat{\boldsymbol{q}}}+\left(\hat{\boldsymbol{K}}-q_{D} \hat{\boldsymbol{E}}_{q}\right) \hat{\boldsymbol{q}}=q_{D} \hat{\boldsymbol{E}}_{u} \boldsymbol{u}+\hat{\boldsymbol{f}}^{A 0}+\hat{\boldsymbol{f}}^{W}
$$

where $\hat{\boldsymbol{q}}$ is the vector of $N_{t}$ nodal degrees of freedom (DOF); $\hat{\boldsymbol{M}}$ and $\hat{\boldsymbol{K}}$ are the associated $N_{t} \times N_{t}$ mass and stiffness matrices; $q_{D}$ is the freestream dynamic pressure; $\hat{\boldsymbol{E}}_{q}$ is a $N_{t} \times N_{t}$ aerodynamic force matrix for the nodal DOF; $\boldsymbol{u}$ is the vector of $N_{u}$ trim variables; $\hat{\boldsymbol{E}}_{u}$ is a $N_{t} \times N_{u}$ matrix giving the steady nodal forces and moments due to unit values of the trim variables; $\hat{\boldsymbol{f}}^{A 0}$ is the nodal aerodynamic load vector due to initial incidence, camber, and twist; and $\hat{f}^{W}$ is the nodal gravity load vector.** Equation (1) includes $N_{r}$ unrestrained rigidbody DOF to represent a free-flying vehicle so that the stiffness matrix has rank $N_{t}-N_{r} . \pm$ The trim condition is assigned by prescribing $N_{u}-N_{r}$ trim variables, hereafter referred to as the fixed trim variables. The vehicle aeroelastic static response and the remaining $N_{r}$ unknown trim variables, hereafter called the free trim variables, are evaluated from Eq. (1) using the linear inertia relief technique $[18,19]$ described in the following.

The total nodal DOF are split into $N_{r}$ reference DOF defining a support frame for the unrestrained rigid-body motions [18] and $N_{l}=N_{t}-N_{r}$ leftover DOF describing the aeroelastic response within the support frame. This is an attached frame with origin at an arbitrary structural grid and orientation fixed with the undeformed configuration, which may not coincide with the orientation of the stability axes [9]. Equation (1) can be partitioned as [9]

$$
\begin{gathered}
{\left[\begin{array}{ll}
\hat{\boldsymbol{M}}_{l l} & \hat{\boldsymbol{M}}_{l r} \\
\hat{\boldsymbol{M}}_{r l} & \hat{\boldsymbol{M}}_{r r}
\end{array}\right]\left\{\begin{array}{l}
\ddot{\hat{\boldsymbol{q}}}_{l} \\
\ddot{\hat{\boldsymbol{q}}}_{r}
\end{array}\right\}+\left[\begin{array}{cc}
\hat{\boldsymbol{K}}_{l l}^{A} & \hat{\boldsymbol{K}}_{l r}^{A} \\
\hat{\boldsymbol{K}}_{r l}^{A} & \hat{\boldsymbol{K}}_{r r}^{A}
\end{array}\right]\left\{\begin{array}{l}
\hat{\boldsymbol{q}}_{l} \\
\hat{\boldsymbol{q}}_{r}
\end{array}\right\}} \\
=-\left[\begin{array}{c}
\hat{\boldsymbol{K}}_{l}^{U} \\
\hat{\boldsymbol{K}}_{r}^{U}
\end{array}\right] \boldsymbol{u}+\left\{\begin{array}{c}
\hat{\boldsymbol{f}}_{l}^{A 0} \\
\hat{\boldsymbol{f}}_{r}^{A 0}
\end{array}\right\}+\left\{\begin{array}{c}
\hat{\boldsymbol{f}}_{l}^{W} \\
\hat{\boldsymbol{f}}_{r}^{W}
\end{array}\right\}
\end{gathered}
$$

where the elements of the vectors $\hat{\boldsymbol{q}}_{l}$ and $\hat{\boldsymbol{q}}_{r}$ are the leftover and reference (support) DOF, respectively; $\hat{\boldsymbol{K}}^{A}:=\hat{\boldsymbol{K}}-q_{D} \hat{\boldsymbol{E}}_{q}$ is the $N_{t} \times$ $N_{t}$ aeroelastic stiffness matrix; and $\hat{\boldsymbol{K}}^{U}:=-q_{D} \hat{\boldsymbol{E}}_{u}$ is a $N_{t} \times N_{u}$ matrix. Since no dynamic structural response occurs at the trim, the accelerations of the leftover DOF can be written as [9]

$$
\ddot{\hat{\boldsymbol{q}}}=\left\{\begin{array}{l}
\ddot{\hat{\boldsymbol{q}}}_{l} \\
\ddot{\hat{\boldsymbol{q}}}_{r}
\end{array}\right\}=\left[\begin{array}{c}
-\hat{\boldsymbol{K}}_{l l}^{-1} \hat{\boldsymbol{K}}_{l r} \\
\boldsymbol{I}
\end{array}\right] \ddot{\hat{\boldsymbol{q}}}_{r}=\boldsymbol{D} \ddot{\hat{\boldsymbol{q}}}_{r}=\boldsymbol{D} \boldsymbol{T}_{r u} \boldsymbol{u}
$$

where $\boldsymbol{D}$ is an $N_{t} \times N_{r}$ matrix describing the rigid-body motion of the structure as a whole due to unit displacements of the reference DOF, while $\boldsymbol{T}_{r u}$ is a $N_{r} \times N_{u}$ matrix giving the reference (support) accelerations from the assigned accelerations of the stability axes. Both matrices are only functions of the model geometry [9].

Substituting Eq. (3) into Eq. (2) and setting $\hat{\boldsymbol{q}}_{r}=0$, the upper partition of Eq. (2) gives

$$
\hat{\boldsymbol{q}}_{l}=\left(\hat{\boldsymbol{K}}_{l l}^{A}\right)^{-1}\left\{\left[\left(\hat{\boldsymbol{M}}_{l l} \hat{\boldsymbol{K}}_{l l}^{-1} \hat{\boldsymbol{K}}_{l r}-\hat{\boldsymbol{M}}_{l r}\right) \boldsymbol{T}_{r u}-\hat{\boldsymbol{K}}_{l}^{U}\right] \boldsymbol{u}+\hat{\boldsymbol{f}}_{l}^{A 0}+\hat{\boldsymbol{f}}_{l}^{W}\right\}
$$

where $N_{r}$ elements of $\boldsymbol{u}$ are still unknown. Once these are evaluated by solving the $N_{r}$ global force/moment equilibrium equations for the unrestrained rigid-body DOF, obtained by premultiplying Eq. (2) by $\boldsymbol{D}^{T}$, Eq. (4) gives the trim aeroelastic response within the support frame.

Note that restraining the reference DOF by setting $\hat{\boldsymbol{q}}_{r}=0$ does not introduce unphysical constraints on the model. Indeed, the vehicle is subjected to a self-balancing load field in the support frame once the apparent inertial loads are taken into account along with the aerodynamic and gravity loads [right-hand side of Eq. (4)]. Computing the elastic response of an unrestrained structure by means

** In the most general case, the vector of trim variables $\boldsymbol{u}=\left\{\alpha, \beta, p^{S}, q^{S}, r^{S}, a_{2}^{S}, a_{3}^{S}, \dot{p}^{S}, \dot{q}^{S}, \dot{r}^{S}, \delta_{a}, \delta_{e}, \delta_{r}\right\}^{T}$ includes the angle of attack $\alpha$; the angle of sideslip $\beta$; the roll, pitch, and yaw angular velocity components of the stability axes denoted by $p^{S}, q^{S}$, and $r^{S}$, respectively; the lateral and vertical linear acceleration components $a_{2}^{S}$ and $a_{3}^{S}$, the angular acceleration components $\dot{p}^{S}, \dot{q}^{S}$, and $\dot{r}^{S}$; and the aileron, elevator, and rudder control-surface rotations denoted by $\delta_{a}, \delta_{e}$, and $\delta_{r}$, respectively. The longitudinal acceleration and thrust input are not considered in linear solutions.

${ }^{\dagger}$ Since the linear analysis does not consider longitudinal translations, one has $N_{r}=2$, vertical translation and pitch rotation, for trim in steady rectilinear flight, and $N_{r}=5$, vertical and lateral translations and all rotations, for trim in steady turn. 
of a restrained analysis using the inertia relief technique is a standard procedure in linear displacement-based FEM computations $[9,18,19]$. However, the inertial relief analysis has never been generalized to nonlinear problems and is not currently implemented in commercial nonlinear FEM solvers like MSC Nastran SOL 400 [12]. This Paper generalizes the inertia relief technique to largeamplitude displacements and applies it to formulate a computationally efficient algorithm for solving nonlinear aeroelastic trim problems using detailed aircraft models.

\section{B. Proposed Nonlinear Algorithm}

The proposed nonlinear aeroelastic trim algorithm is oriented to the coupling of off-the-shelf structural and aerodynamic solvers to analyze very flexible aircraft described by generic models, including high-fidelity representations. In the context of this Paper, the algorithm is implemented by coupling MSC Nastran SOL 400 [12] with an in-house VLM code [14], but the methodology is applicable to any off-the-shelf solver.

The nonlinear aeroelastic trim problem is solved iteratively by fixing an arbitrary reference grid in order to have a nonsingular model stiffness matrix at each iteration. As such, structural displacements are evaluated with respect to a reference grid as in the linear algorithm described in Sec. II.A. To simulate the vehicle as unrestrained, the apparent inertia load experienced in the support frame is evaluated at each iteration using a novel large-amplitude inertia relief technique [11] that generalizes the linear technique of Sec. II.A. The apparent inertia load is then applied in combination with the aerodynamic and gravity loads in order to obtain an overall self-balancing distribution in the support frame. As a result, the displacement field obtained at each iteration is representative of an unrestrained structure, although computed by means of a restrained analysis. In contrast, fixing an arbitrary grid without adding the apparent inertial load would give a nonzero reactive force at the constrained structural point. For a steady rectilinear flight condition, the reaction would eventually vanish at convergence but would still be present during the solution process giving an unphysical load path to the trim solution. Additionally, neglecting the apparent inertial load would be not correct for trimming maneuvers.
The proposed algorithm is illustrated in Fig. 1 and consists of an outer and a nested Newton-Raphson loop. Starting from a first guess, the outer Newton-Raphson loop updates the $N_{r}$ free trim variables based on their current values, system Jacobian matrix, and force/ moment resultants in order to trim the vehicle in the prescribed flight condition. The force/moment resultants are evaluated by the nested Newton-Raphson loop, which computes the nonlinear aeroelastic static response for the current set of trim variables.

The nested fluid-structure loop uses the method of [20] to closely couple the aerodynamic and structural solvers along with a novel large-amplitude inertia relief technique to simulate the vehicle as unrestrained at each iteration. The generic $j$ th iteration of the nested loop is performed by first calling on the nonlinear aerodynamic solver to compute the steady aerodynamic load on the current deformed configuration, which is transferred to the structural grids. In the present Paper, the load transfer is realized using six DOF force splines in order to consider all the displacement and rotation components at each aerodynamic and structural node $[9,10]$. Next, the inertia relief load experienced in the support frame due to the nonbalanced force/ moment resultants of aerodynamics and gravity along the reference (support) DOF is computed and given as input to the structural solver along with the aerodynamic and gravity load. The structural solver then computes the updated displacements of the leftover DOF (with the reference DOF constrained) by solving the nonlinear system

$$
\hat{\boldsymbol{K}}_{l l}^{(j)} \hat{\boldsymbol{q}}_{l}^{(j+1)}=s_{j}\left(\hat{\boldsymbol{f}}_{l}^{A(j)}+\hat{\boldsymbol{f}}_{l}^{W}+\hat{\boldsymbol{f}}_{l}^{I(j)}\right)
$$

where $\hat{\boldsymbol{K}}_{l l}^{(j)}$ is the stiffness matrix evaluated at the $j$ th iteration and the previous solution $\hat{\boldsymbol{q}}_{l}^{(j)}$ is assumed as the first guess. The forcing vector on the right-hand side of Eq. (5) is the total load experienced in the support frame, $\hat{\boldsymbol{f}}_{l}^{A(j)}$ being the aerodynamic load, $\hat{\boldsymbol{f}}_{l}^{I(j)}$ being the inertia relief load, and $s_{j} \leq 1$ being a scaling factor to perform an incremental-load relaxation (see Sec. I). Note that not only the aerodynamic load but also the inertia relief load is evaluated on the last deformed configuration and updated in the loop, as necessary in the presence of large deflections (see Sec. II).

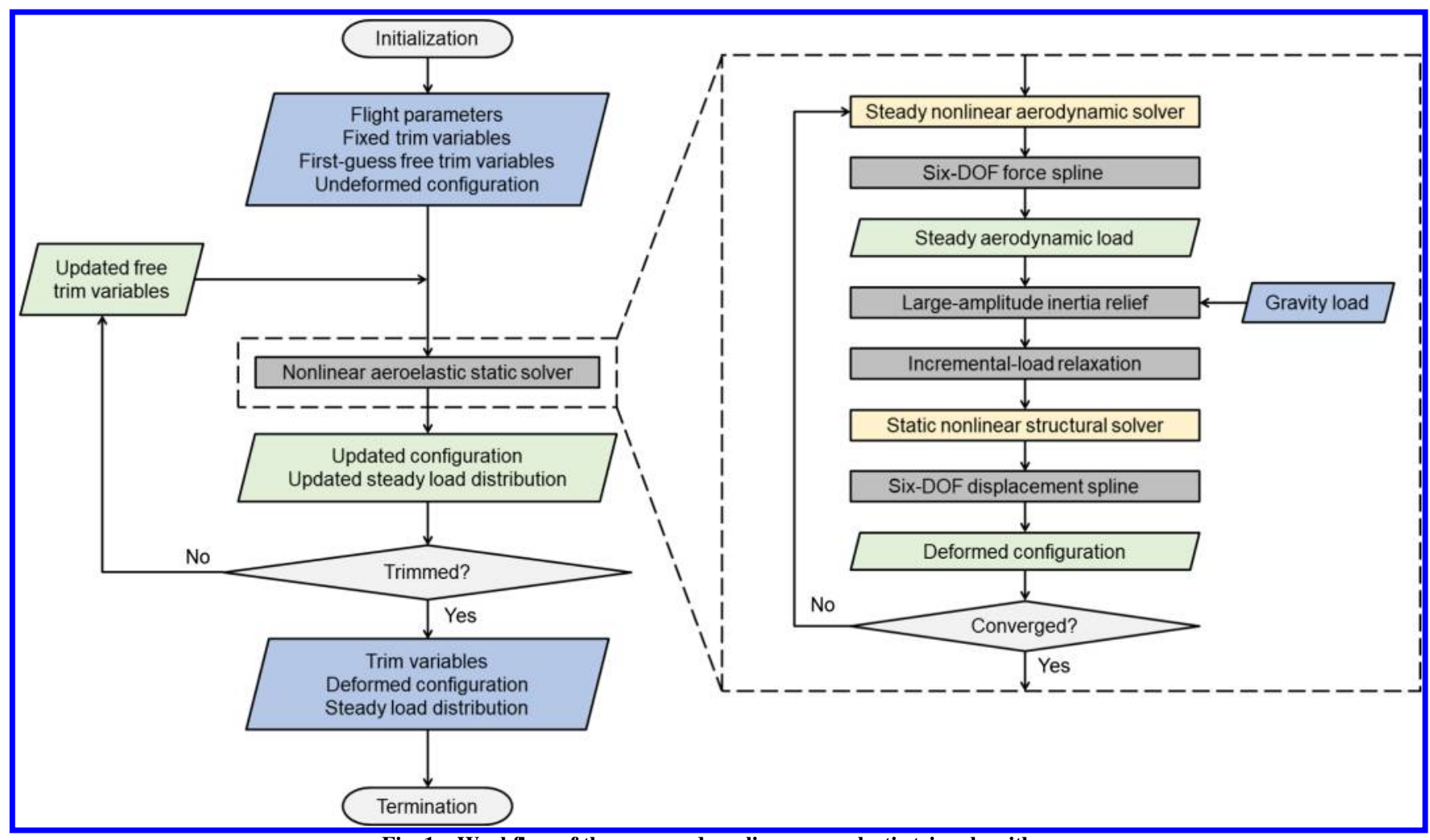

Fig. 1 Workflow of the proposed nonlinear aeroelastic trim algorithm. 
Once Eq. (5) is solved, the incremental displacement $\Delta \hat{\boldsymbol{q}}_{l}^{(j)}=$ $\hat{\boldsymbol{q}}_{l}^{(j+1)}-\hat{\boldsymbol{q}}_{l}^{(j)}$ is interpolated to update the aerodynamic model and, in the case of CFD models, deform the fluid-domain volume mesh. In the present Paper, the displacement interpolation is performed using six-DOF splines $[9,10]$. The $j$ th fluid-structure iteration is completed by checking the convergence of structural displacements. If the convergence is reached, the solution is returned to the outer loop to verify if the trim condition is satisfied; otherwise, a new fluidstructure iteration is performed.

The incremental-load relaxation procedure used to closely couple the aerodynamic and structural solvers is briefly described in the following, followed by the proposed large-amplitude inertia relief technique that is the original contribution from the present work.

\section{Incremental-Load Relaxation}

In nonlinear aeroelastic static analyses, the structure equilibrium must be satisfied in the deformed configuration, which requires an iterative solution process involving staggered calls to the aerodynamic and structural solvers (see Fig. 1). If incremental deflections are large, the aerodynamic load applied to the structure at the $j$ th iteration may be inconsistent with the updated model geometry, which negatively impacts the solution stability, accuracy, and computational cost. The same holds for the inertia relief load, which is also function of structural displacements as discussed in Sec. II.

To ensure the consistency of applied loads and structural displacements, the present algorithm uses an incremental-load relaxation procedure [20] to closely couple the aerodynamic and structural solvers. The structure is loaded incrementally in $n_{s}$ iterations of the $j$ loop, which corresponds to a load-step size $1 / n_{s}$. Each nonlinear structural analysis assumes the last deformed configuration as the first guess and takes into account the differential stiffness. The load field applied at a generic iteration $j \leq n_{s}$ is multiplied by a scaling factor $s_{j}=j / n_{s}\left(j=1, \ldots, n_{s}\right)$ [see Eq. (5)]. As such, only a load percentage $s_{1}=1 / n_{s}$ is applied to the structure at the first iteration, so limiting incremental displacements. At the second iteration, the load field computed on the updated geometry and scaled by $s_{2}=2 / n_{s}$ replaces the previous distribution, and the procedure is repeated until a unit scaling factor is achieved after $n_{s}$ iterations. At this point, the structure is fully loaded, but additional $n_{a}$ fluid-structure iterations may be still necessary for convergence. These are performed with the same load updating approach but assuming $s_{j}=1$ for any $j>n_{s}$. The total number of iterations for a complete analysis is thus $n_{t}=n_{s}+n_{a}$, given by $n_{s}$ load-step iterations and $n_{a}$ additional iterations to get convergence once the structure is fully loaded.

For appropriate choices of the number of load steps, the incremental-load relaxation improves the algorithm stability, accuracy, and computational speed. Using multiple load steps reduces incremental displacements between subsequent calls to the structural solver, which facilitates the convergence of each nonlinear static analysis and allows one to better simulate follower loads [20]. The load relaxation also helps the convergence of the aerodynamic solver and improves the aerodynamic model updating, especially when using CFD aerodynamics that requires fluid-domain volume mesh deformation. On the other hand, performing too many load steps increases computational time with no benefic effect on the solution. As such, a preliminary sensitivity analysis is necessary to tailor the load-step size to the expected level of problem nonlinearity.

\section{Large-Amplitude Inertia Relief}

Conventional displacement-based FEM static analysis cannot be performed on unrestrained structures due to the stiffness matrix singularity. However, commercial FEM solvers like MSC Nastran use the inertia relief technique to simulate unrestrained structures in linear structural and aeroelastic analyses $[\underline{9}, 18,19]$.

The basic principle of the inertia relief is that a free-free structure subjected to a nonbalanced load field experiences a rigid-body acceleration. Therefore, structural analyses conducted within a noninertial reference frame fixed with the accelerated structure (support frame) must include the apparent inertial loads due to such a rigid-body acceleration state. Once the apparent inertial loads are applied in combination with the external loads, the structure is subjected to a self-balancing load distribution in the support frame. This allows one to compute the elastic displacement of the unrestrained structure via a restrained analysis performed by fixing a reference structural grid. Indeed, no constraint is actually placed on the model, since the reactive load at the reference grid is zero. As such, structural responses obtained with different choices of the reference grid (support frame) differ for a rigid-body displacement and describe the unique deformed state of the unrestrained structure subjected to the original nonbalanced external load [21].

Unfortunately, the inertia relief analysis has never been generalized to large displacements and is not currently implemented in commercial nonlinear FEM solvers $[12,13]$. Therefore, there is no built-in algorithm to include the apparent inertial loads on the righthand side of Eq. (5). In the case of trim in steady rectilinear flight, aerodynamics and weight are self-balancing at convergence, so results obtained without including the apparent inertial loads in the loop are still representative of an unrestrained structure. However, this is not valid when trimming maneuvers. Additionally, even when trimming steady rectilinear flight conditions, the apparent inertial load is not zero in the intermediate loop iterations. Neglecting to include this load gives an unphysical load path to the trim solution, which may negatively impact the stability, accuracy, and computational cost of the analysis.

In the proposed algorithm, the apparent inertial loads due to the nonbalanced force/moment resultants of aerodynamics and gravity are taken into account at each iteration to simulate the vehicle as unrestrained in the whole solution process. The inertia relief load field is computed by fixing a generic reference structural grid to define a support frame. Generalizing the MSC Nastran linear inertia relief technique [19], the apparent inertial load to obtain an overall self-balancing load distribution in the support frame at the current iteration is evaluated as

$$
\hat{\boldsymbol{f}}^{I(j)}=-\left.\hat{\boldsymbol{M}} \boldsymbol{D} \ddot{\hat{\boldsymbol{q}}}_{r}\right|_{(j)}=-\hat{\boldsymbol{M}} \boldsymbol{D}^{(j)}\left[\boldsymbol{D}^{(j) T} \hat{\boldsymbol{M}} \boldsymbol{D}^{(j)}\right]^{-1} \boldsymbol{D}^{(j) T}\left(\hat{\boldsymbol{f}}^{A(j)}+\hat{\boldsymbol{f}}^{W}\right)
$$

where $\boldsymbol{D}^{(j)}$ is the rigid-body mode matrix of Eq. (3) evaluated by considering the deformed model configuration at the $j$ th iteration. The dependency of the inertia relief load on structural displacements is neglected in the linear inertia relief analysis $[18,19]$, whereas it must be taken into account in the presence of large deflections.

The inertia relief load in Eq. (6) is the apparent inertial load experienced in the support frame due to the structure rigid-body acceleration state caused by the nonbalanced force/moment resultants of aerodynamics and gravity along the reference DOF. The partition related to the leftover DOF is added to the right-hand side of Eq. (5) to simulate the structure as unrestrained in up to $N_{r} \leq 6$ rigid-body DOF. This approach can be applied to a generic nonlinear displacement-based FEM analysis, not necessarily a nonlinear aeroelastic trim analysis.

The proposed large-amplitude inertia relief technique allows one to reach the trim solution by following a physical load path simulating the structure as unrestrained at each loop iteration. As shown in Sec. IV.C, this improves the convergence smoothness and speed compared to fixing a structural grid without including the apparent inertial loads. Indeed, although the loop iterations of a nonlinear static analysis do not have the physical meaning of time steps, the largeamplitude inertia relief combined with the incremental-load relaxation procedure numerically simulates the gradual achievement of the trim deformed configuration actually experienced by a freeflying flexible vehicle.

\section{Computational Framework and Comparison with Existing Algorithms}

The proposed algorithm is implemented in a medium-fidelity computational environment coupling MSC Nastran SOL 400 [12] 
Table 1 Features of nonlinear aeroelastic trim solvers

\begin{tabular}{lccc}
\hline \hline Feature & Present solver & UM/NAST solver & DLR solver \\
\hline Structural model & Any FEM model & Strain-based beams & Any FEM model \\
Aerodynamic model & VLM & VLM & VLM \\
Coupling approach & Six-DOF splines & Strip based & RBF \\
Relaxation & Yes & No & No \\
Inertia relief & Yes & Not applicable & No \\
\hline \hline
\end{tabular}

with a in-house nonlinear VLM solver developed at DLR [14], which neglects wake roll-up effects but allows for large displacements and rotations of the aerodynamic grids. The framework is validated by comparing results with UM/NAST [6] and the DLR aeroelastic toolbox [14]. The main features of these environments are summarized in the following and in Table 1 .

UM/NAST models nonlinear structures using a strain-based geometrically exact beam formulation []. Trim solutions for complete vehicles are obtained by either solving the force/moment equilibrium or forcing zero linear and angular accelerations at the origin of the body reference frame. The trim solver consists of an outer Newton-Raphson loop on the trim variables and a nested fluidstructure loop that couples the strain-based beam formulation with strip theory, surrogate models, or, more recently, with the VLM code from DLR [14]. Fluid-structure coupling is performed by transferring the load distribution on each aerodynamic strip to the corresponding beam axis location and by recovering the aerodynamic cross-section displacement from the beam axis bending and torsion. Inertia relief is not used in UM/NAST since the strain-based model stiffness matrix is always definite positive.

The DLR aeroelastic toolbox is a simulation environment for aircraft described by generic FEM models that includes linear, reduced-order, or fully nonlinear structural formulations [14]. The nonlinear aeroelastic trim solver consists of a main Newton-Raphson loop and a nested fluid-structure loop coupling MSC Nastran SOL 400 with the nonlinear VLM code that was provided for developing the present environment and for coupling with UM/NAST [22]. Aerodynamic and structural grids are coupled using the radial basis function (RBF) technique and, for beam-type FEM models, by developing a rigid-cross-section coupling model to interface onedimensional structures and two-dimensional lifting surfaces. Incremental-load relaxation is not implemented in the loop. A structural grid is clamped to eliminate the stiffness matrix singularity, but the inertia relief load is not taken into account.

\section{Numerical Results}

This section discusses numerical case studies to validate the developed computational framework and investigate the effects of the proposed large-amplitude inertia relief technique on nonlinear aeroelastic trim solutions. All the analyses are conducted using MSC Nastran beam-type models in order to directly compare the results with UM/NAST []].

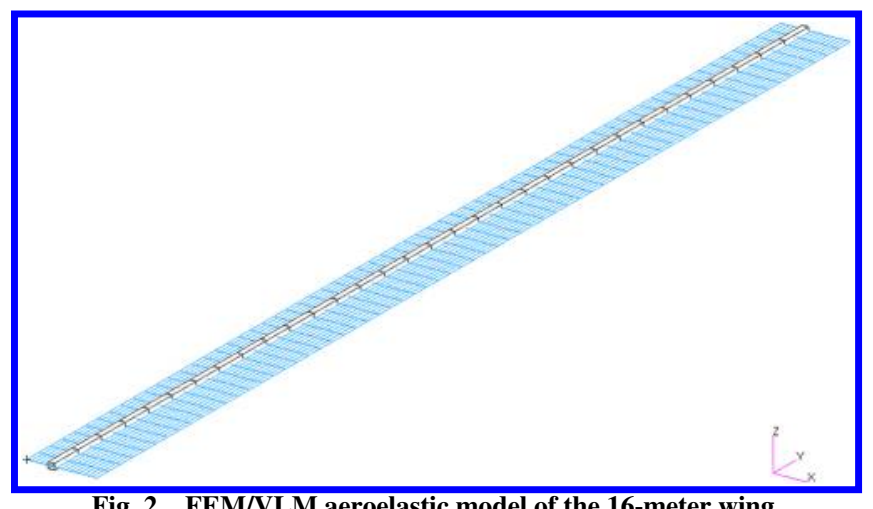

Fig. 2 FEM/VLM aeroelastic model of the 16-meter wing.
Table 2 Nonlinear aeroelastic static response of the $16 \mathrm{~m}$ wing: sensitivity to the step size for $\alpha=5 \mathrm{deg}$

\begin{tabular}{lcrrrr}
\hline \hline Step size, $\%$ & $\bar{u}_{z_{\text {tip }}}, \%$ & $\Delta t, \%$ & $n_{s}$ & $n_{a}$ & $n_{t}$ \\
\hline 100 & 20.31 & -- & 1 & 4 & 5 \\
50 & 20.26 & 40.57 & 2 & 4 & 6 \\
20 & 20.21 & 64.60 & 5 & 4 & 9 \\
10 & 20.20 & 110.33 & 10 & 3 & 13 \\
5 & 20.19 & 308.57 & 20 & 3 & 23 \\
\hline \hline
\end{tabular}

\section{A. Validation of Nonlinear Aeroelastic Static Solver}

The core module coupling MSC Nastran SOL 400 with the VLM code is validated by analyzing the nonlinear aeroelastic static response of a highly flexible $16 \mathrm{~m}$ wing [23] and of the X-HALE risk reduction vehicle (RRV) [24] at a prescribed freestream velocity and angle of attack. The analyses are performed by assuming clamped boundary conditions at the root for the wing and at the wing centerline for the $\mathrm{X}$-HALE. As such, the inertial relief module is not active at this stage.

\section{Highly Flexible Wing}

The FEM/VLM aeroelastic model of the highly flexible $16 \mathrm{~m}$ wing [23] is shown in Fig. 2. The MSC Nastran structural model consists of a beam-type structure with stiffness properties that vary quadratically along the span and lumped masses with concentrated inertia tensors to tune the dynamic behavior on the reference UM/NAST model. The VLM aerodynamic model consists of a rectangular flat-plate lifting surface. In this Paper, aerodynamic and structural grids are coupled using 16 six-DOF finite beam spline patches.

The nonlinear aeroelastic static response of the $16 \mathrm{~m}$ wing is studied for incompressible flow, $\rho_{\infty}=1.225 \mathrm{~kg} / \mathrm{m}^{3}, V_{\infty}=40 \mathrm{~m} / \mathrm{s}, \alpha=3$, 4, $5 \mathrm{deg}$, and by neglecting weight. The results are compared with solutions from 1) the UM/NAST solver based on strip-theory aerodynamics corrected with weighting factors [23], 2) the UM/NAST solver based on VLM aerodynamics [22], and 3) the MSC Nastran SOL 400/VLM solver of the DLR toolbox [14].

The sensitivity to the load-step size used in the incremental-load relaxation is preliminarily analyzed for $\alpha=5 \mathrm{deg}$. Table 2 shows the converged wing-tip vertical displacement normalized by the span $\bar{u}_{z_{\text {tip }}}$; the relative variation in the computational time with respect to the no-relaxation case; and the number of load-step, additional, and total iterations for convergence. The wing-tip vertical displacement decreases with the step size due to the more accurate simulation of follower forces that better captures the wing shortening, but no practical variation is found for a step size below $10 \%$. As such, this value is assumed for all the examined angles of attack. The computational time increases for a smaller step size due to the increase in the total number of iterations. This is because the analyses converge very fast even with no relaxation for this simple test case. Therefore, increasing the number of load steps improves the solution accuracy but results in a slower convergence since the aerodynamic load is applied in increments.

The converged wing-tip vertical displacements normalized by the span obtained with different approaches are compared in Table $\underline{3}$. The deformed configurations are plotted in Fig. 3 . The present results match those from UM/NAST with VLM aerodynamics for all the examined conditions. The DLR toolbox solution practically lies on 
Table 3 Nonlinear aeroelastic static response of the $16 \mathrm{~m}$ wing: wing-tip vertical displacement

\begin{tabular}{lccr}
\hline \hline Methodology & \multicolumn{3}{c}{$\bar{u}_{\text {zip }_{\text {ip }}}, \%(\alpha=3,4,5 \mathrm{deg})$} \\
\hline Proposed algorithm & 12.60 & 16.51 & 20.20 \\
UM/NAST with VLM & 12.60 & 16.50 & 20.18 \\
UM/NAST with strip theory & 12.36 & 16.18 & 19.78 \\
DLR toolbox & 12.62 & 16.57 & 20.33 \\
\hline \hline
\end{tabular}

top of the previous ones, with a maximum wing-tip difference of $0.65 \%$ at $\alpha=5 \mathrm{deg}$. The deflections from UM/NAST with strip theory are smaller than those obtained considering VLM aerodynamics, with a relative wing-tip difference that increases from 1.85 to $2 \%$ with the angle of attack. This behavior is motivated by considering that the weighting factors used to correct the strip-theory aerodynamic load distribution are evaluated on the rigid undeformed configuration [23].

\section{X-HALE RRV}

The FEM/VLM aeroelastic model of the X-HALE RRV [24] is shown in Fig. 4. The MSC Nastran structural model consists of beamtype members for the wing elastic axis; rigid bars for the pods, fins, tail booms, and horizontal/vertical tails; and lumped-mass elements with concentrated inertia tensors to tune the dynamic behavior on the UM/NAST model. The VLM aerodynamic model consists of a cambered lifting surface for the wing with the EMX07 airfoil at $5 \mathrm{deg}$ incidence [15], while pods, fins, and tails are modeled as flat plates.

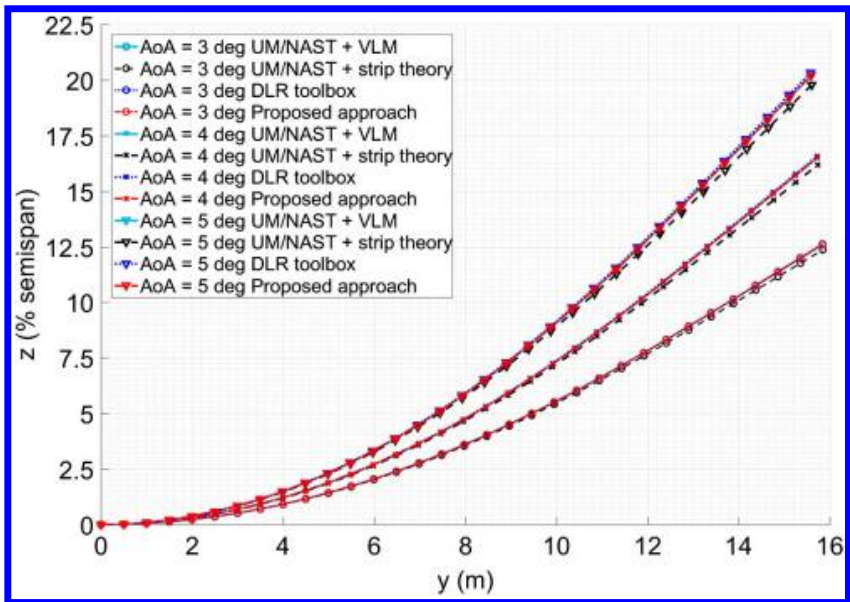

Fig. 3 Nonlinear aeroelastic static response of the $16 \mathrm{~m}$ wing: deformed configuration.

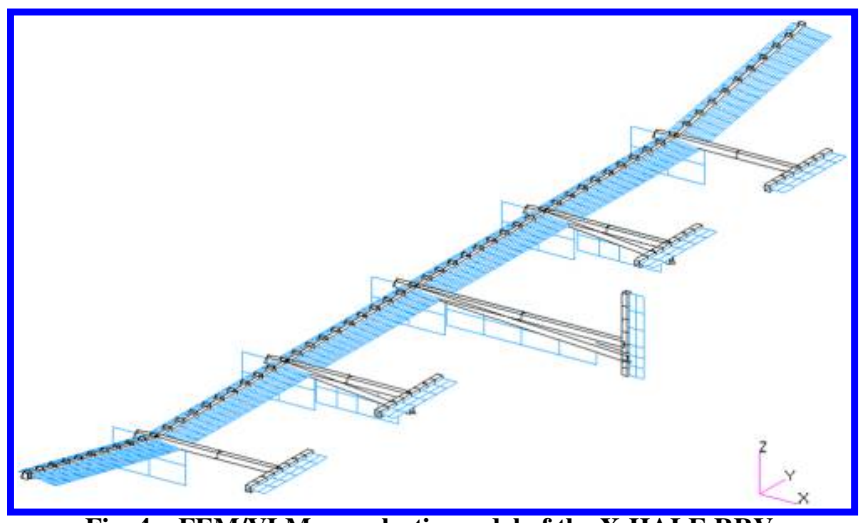

Fig. 4 FEM/VLM aeroelastic model of the X-HALE RRV.
Table 4 Nonlinear aeroelastic static response of the X-HALE RRV: sensitivity to the step size for $\alpha=1 \mathrm{deg}$

\begin{tabular}{lccrrr}
\hline \hline Step size, $\%$ & $\bar{u}_{z_{\text {tip }}}, \%$ & $\Delta t, \%$ & $n_{s}$ & $n_{a}$ & $n_{t}$ \\
\hline 100 & 17.43 & -- & 1 & 28 & 29 \\
50 & 16.79 & -33.52 & 2 & 20 & 22 \\
20 & 16.41 & -26.14 & 5 & 19 & 24 \\
10 & 16.30 & -14.85 & 10 & 17 & 27 \\
5 & 16.24 & 12.16 & 20 & 15 & 35 \\
\hline \hline
\end{tabular}

Table 5 Nonlinear aeroelastic static response of the X-HALE RRV: right wing-tip vertical displacement

\begin{tabular}{lccc}
\hline \hline Methodology & \multicolumn{3}{c}{$\bar{u}_{z_{\text {tip }}}, \%(\alpha=0,0.5,1 \mathrm{deg})$} \\
\hline Proposed algorithm & 11.74 & 14.07 & 16.30 \\
UM/NAST with VLM & 11.88 & 14.40 & 16.81 \\
DLR toolbox & 11.69 & 14.05 & 16.31 \\
\hline \hline
\end{tabular}

In this Paper, aerodynamic and structural grids are coupled using 20 six-DOF finite beam spline patches for the wing and a single patch for every other lifting surface.

The nonlinear aeroelastic static response of the X-HALE RRV is studied for incompressible flow, $\rho_{\infty}=1.222 \mathrm{~kg} / \mathrm{m}^{3}, V_{\infty}=16 \mathrm{~m} / \mathrm{s}$, $\alpha=0,0.5,1 \mathrm{deg}$, and by taking into account weight. The results are compared with solutions from 1) the UM/NAST solver based on VLM aerodynamics [22] and 2) the MSC Nastran SOL 400/VLM solver of the DLR toolbox [14].

The sensitivity to the load-step size is preliminarily analyzed for $\alpha=1 \mathrm{deg}$ as reported in Table 4 . In contrast with the case of the $16 \mathrm{~m}$ wing, computational time does not increase monotonically by reducing the step size for the X-HALE RRV. Indeed, it first decreases by $33 \%$ when reducing the step size from 100 to $50 \%$; next, it increases again for further step-size reductions. However, the convergence using ten load steps is still $15 \%$ faster than in the no-relaxation case. This is because $n_{s}$ increases for a smaller step size, while $n_{a}$ decreases due to the smoother convergence to the deformed configuration. The former effect is dominant for the $16 \mathrm{~m}$ wing, since the analysis converges very fast even with no relaxation and $n_{a}$ is not significantly affected by the number of load steps (see Table 2). In contrast, $n_{a}$ significantly decreases from 28 to 15 for the X-HALE when the step size is reduced from 100 to $5 \%$. Since the converged solution does not practically vary for step size below $10 \%$, this value is assumed for all the angles of attack.

The converged results obtained with different approaches for $\alpha=0,0.5,1 \mathrm{deg}$ are compared in Table 5. The deformed configurations are illustrated in Fig. 5. The present results match the ones from the DLR toolbox for all the flight conditions. The UM/NAST solutions show larger displacements, with a wing-tip difference that increases from 1.1 to $3 \%$ with the angle of attack. This behavior is justified by considering that in UM/NAST the aerodynamic load transferred to each structural grid is obtained by interpolating the force and moment resultants on the two closest aerodynamic strips. As such, each structural grid is aerodinamically influenced only by VLM panels in a near neighborhood. The sixDOF splines used in the present framework and the RBF technique used in the DLR toolbox are implemented by coupling each structural node with a larger region of the aerodynamic model. As such, the load transferred to each structural node is influenced by multiple aerodynamic cross-sections. The different coupling strategies do not influence the results for the $16 \mathrm{~m}$ wing, while a higher sensitivity to the size of the coupled aerodynamic and structural regions is expected for more complex configurations like X-HALE. 


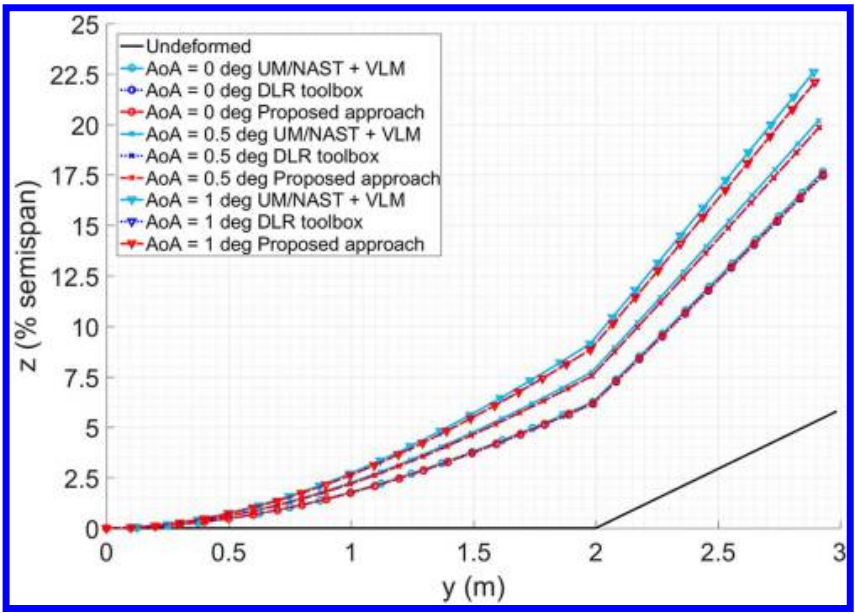

Fig. 5 Nonlinear aeroelastic static response of the X-HALE RRV: right half-wing deformed configuration.

\section{B. Validation of Nonlinear Aeroelastic Trim Solver}

The whole nonlinear aeroelastic trim solver is validated by trimming the X-HALE RRV for steady rectilinear flight in incompressible flow at $\rho_{\infty}=1.222 \mathrm{~kg} / \mathrm{m}^{3}$ and $V_{\infty}=16 \mathrm{~m} / \mathrm{s}$, which is a typical operating condition [16]. The analysis is conducted using the horizontal tails as elevators. The results are compared with solutions from 1) the UM/NAST solver based on VLM aerodynamics [22], 2) the MSC Nastran SOL 400/VLM solver of the DLR toolbox [14], and 3) the linear solver MSC Nastran SOL 144 [9]. The inertia relief module is activated at this stage to simulate free-free boundary conditions at each iteration. The reference grid defining the support frame is chosen at the wing centerline in order to directly compare the trim deflection with the results from UM/NAST and the DLR toolbox. The analysis is performed using a step size equal to $20 \%$ [24], for which the solution converges $10 \%$ faster than with no relaxation.

A linear VLM model based on the nonlinear one is developed for conducting the aeroelastic trim analyses using MSC Nastran SOL 144 [9]. Since the linear VLM formulation does not account for incidence, camber, and twist effects [9], a static correction is developed for the X-HALE wing using the downwash input method [9] and a direct load input [11]. The downwash input method is a corrective technique available in MSC Nastran SOL 144 that allows one to adjust the linearized aerodynamic boundary conditions by taking into account the static rotation of panel normal vectors due to incidence, camber, and twist. The direct load input is implemented by including the aerodynamic load field evaluated on the rigid configuration at $V_{\infty}=16 \mathrm{~m} / \mathrm{s}, \alpha=0 \mathrm{deg}$, and $\delta_{e}=0 \mathrm{deg}$ using the nonlinear VLM code as an external load distribution in the linear aeroelastic trim analysis.

The results obtained with different approaches are reported in Table 6 . The right half-wing deformed configurations are compared in Fig. 6. The present solver and the DLR toolbox show a better agreement in terms of deformed configuration and elevator rotation, whereas the UM/NAST and DLR toolbox trim angles of attack are closer. However, differences between UM/NAST and the other solutions are expected due to the local coupling approach (see

Table 6 Nonlinear aeroelastic trim of the X-HALE RRV: right wing-tip vertical displacement and trim variables

\begin{tabular}{lccc}
\hline \hline & $\bar{u}_{z_{\text {tip }}}, \%$ & $\alpha_{e}, \operatorname{deg}$ & $\delta_{e_{e}}$, deg \\
\hline Proposed algorithm & 5.07 & 0.75 & 2.42 \\
UM/NAST with VLM & 4.70 & 0.79 & 2.62 \\
DLR toolbox & 5.00 & 0.79 & 2.47 \\
MSC Nastran SOL 144 with load input & 4.90 & 0.73 & 2.60 \\
MSC Nastran SOL 144 with downwash input & 4.61 & 0.72 & 2.61 \\
\hline \hline
\end{tabular}

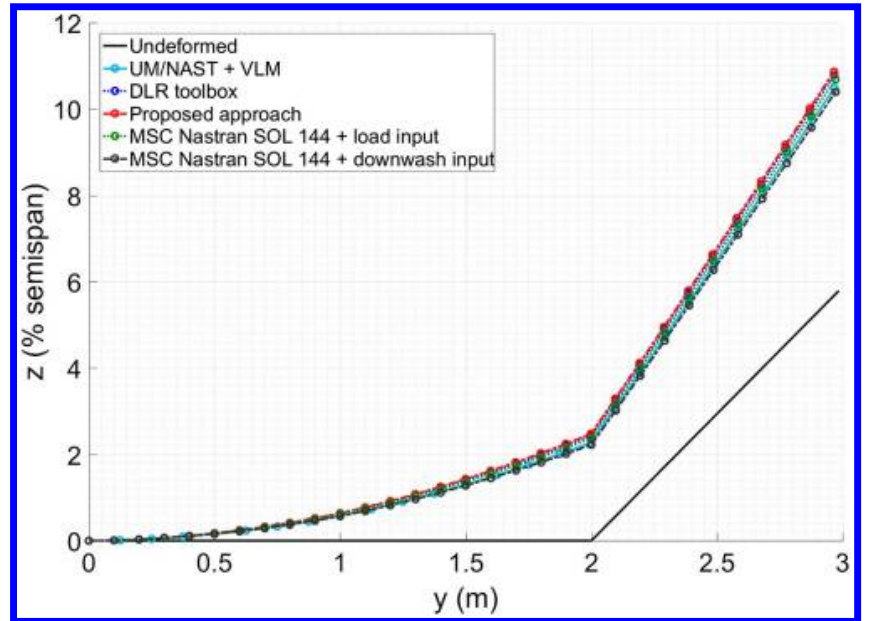

Fig. 6 Nonlinear aeroelastic trim of the X-HALE RRV: right-half wing deformed configuration.

Sec. II). Since the X-HALE RRV deflection is moderate at the examined trim point, the nonlinear and linear results are also in good agreement. The linear results obtained using the direct load input are closer to the nonlinear solution since the corrective external load is evaluated on the actual VLM model geometry considering the wing camber and incidence.

\section{Effect of Inertia Relief}

Having validated the computational framework, X-HALE is trimmed for steady rectilinear flight at $V_{\infty}=15,16,17,18 \mathrm{~m} / \mathrm{s}$ with and without using the large-amplitude inertia relief technique to investigate its effect on the solution process. The structure is simulated as constrained in the loop when the inertia relief is off, since the apparent inertial loads are not taken into account and aerodynamics and gravity give a self-balancing load distribution in the support frame only when the trim condition is satisfied (last iteration). Conversely, the structure is simulated as unrestrained during the whole solution process when using the inertia relief, since the apparent inertial loads are added to aerodynamics and gravity at each iteration. As such, the cases of inertia relief off/on correspond to reaching the trim condition by following load paths with different boundary conditions in the loop.

The trim results in terms of right wing-tip vertical displacement normalized by the half-span, angle of attack, elevator rotations, relative variation in the computational time, and number of iterations with inertia relief off/on are reported in Table 7. The convergence histories of the right wing tip are plotted in Fig. 7. All the analyses are performed using a load-step size equal to $20 \%$. Figure 7 shows that the proposed large-amplitude inertia relief technique eliminates large numerical oscillations around the trim deformed configuration and dramatically reduces computational time for all the examined flight conditions (see Table 7). The results with inertia relief off/on are slightly different (see Table 7) since the model stiffness matrix is updated in the loop and is thus sensitive to the load path.

Table 7 Nonlinear aeroelastic trim of the X-HALE RRV: trim results with inertia relief off/on

\begin{tabular}{lcccccc}
\hline \hline$V_{\infty}, \mathrm{m} / \mathrm{s}$ & Inertia relief & $\bar{u}_{z_{\text {tip }}}, \%$ & $\alpha_{e}, \operatorname{deg}$ & $\delta_{e_{e}}, \operatorname{deg}$ & $\Delta t, \%$ & $n_{t}$ \\
\hline 15 & Off & 5.29 & 1.52 & 1.85 & -- & 75 \\
15 & On & 5.27 & 1.53 & 1.84 & -49.91 & 32 \\
16 & Off & 5.19 & 0.72 & 2.44 & -- & 87 \\
16 & On & 5.07 & 0.75 & 2.42 & -55.75 & 32 \\
17 & Off & 4.95 & 0.09 & 2.89 & -- & 99 \\
17 & On & 4.86 & 0.11 & 2.87 & -55.36 & 38 \\
18 & Off & 5.23 & -0.54 & 3.34 & -- & 140 \\
18 & On & 4.66 & -0.43 & 3.23 & -69.02 & 40 \\
\hline \hline
\end{tabular}



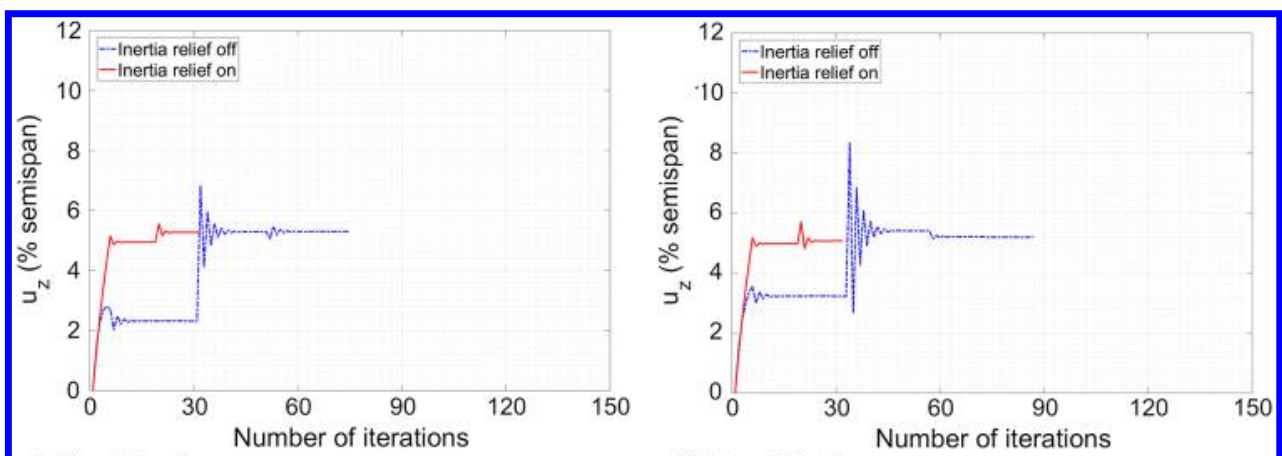

a) $V_{\infty}=15 \mathrm{~m} / \mathrm{s}$

b) $V_{\infty}=16 \mathrm{~m} / \mathrm{s}$
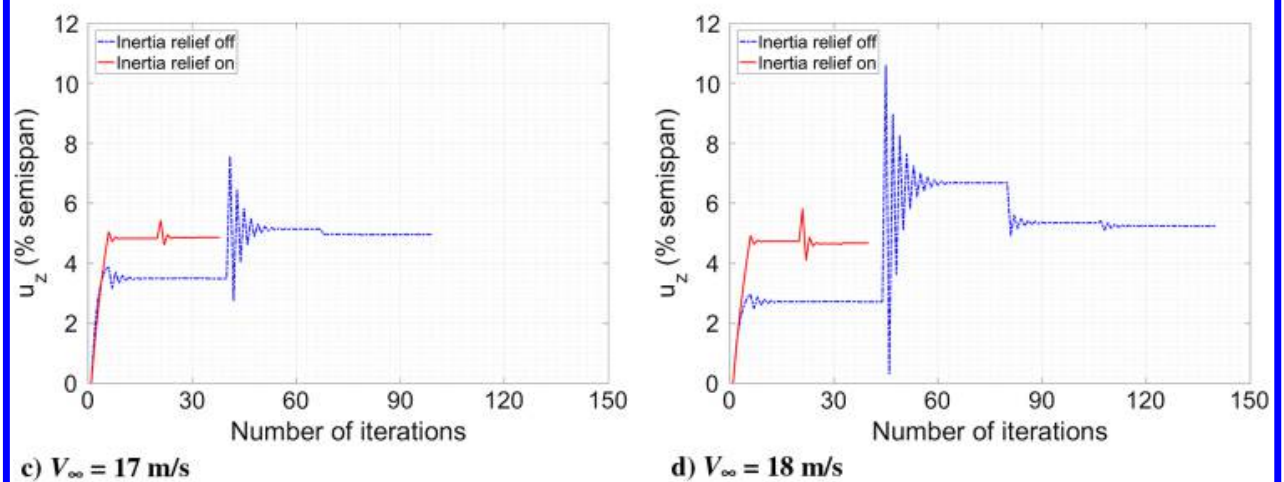

Fig. 7 Nonlinear aeroelastic trim of the X-HALE RRV: right wing-tip vertical displacement convergence history with inertia relief off/on.

\section{Conclusions}

This Paper presented a computationally efficient algorithm for the nonlinear aeroelastic trim analysis of very flexible aircraft described by models of any fidelity. The algorithm is based on a novel largeamplitude inertia relief technique and is applicable to fluid-structure interaction frameworks coupling generic structural and aerodynamic solvers, including high-fidelity commercial solvers.

The methodology was tested on a low-order model of the University of Michigan's X-HALE in order to compare it to reference results for a typical steady rectilinear flight condition. Having validated the computational framework, the benefits of the proposed approach were demonstrated by trimming the vehicle at different flight speeds with and without using the large-amplitude inertia relief. Including the apparent inertial loads at each iteration in the loop resulted in a physical load path to the trim solution giving a smoother and faster convergence compared to fixing an arbitrary grid without using the inertia relief.

High-fidelity nonlinear aeroelastic design of next-generation aircraft is inherently a computationally expensive process requiring efficient and robust algorithms to ensure analysis convergence in acceptable times and for large design spaces. Despite a low-order model being considered in this Paper for validation purposes, the results demonstrated that the proposed nonlinear aeroelastic trim algorithm has the potential for application to high-fidelity design. Future work will apply the methodology to very flexible configurations described by built-up structural and aerodynamic models, which are the ultimate target of the proposed approach.

\section{Acknowledgments}

The authors would like to thank the Ph.D. Candidates Patricia Teixeira and Ryan Kitson (University of Michigan's Active Aeroelasticity and Structures Research Laboratory) for providing the University of Michigan's Nonlinear Aeroelastic Simulation Toolbox results. The first author would like to thank the Ph.D. program of Sapienza University of Rome for funding the research on nonlinear aeroelastic trim conducted as a short-term visiting scholar at the University of Michigan.

\section{References}

[1] Cesnik, C. E. S., Palacios, R., and Reichenbach, E. Y., "Reexamined Structural Design Procedures for Very Flexible Aircraft," Journal of Aircraft, Vol. 51, No. 5, 2014, pp. 1580-1591. doi:10.2514/1.C032464

[2] Patil, M. J., and Hodges, D. H., "Flight Dynamics of Highly Flexible Flying Wings," Journal of Aircraft, Vol. 43, No. 6, 2006, pp. 1790-1799. doi: $10.2514 / 1.17640$

[3] Raghavan, B., and Patil, M. J., "Flight Dynamics of High Aspect-Ratio Flying Wings: Effect of Large Trim Deformation," Journal of Aircraft, Vol. 46, No. 5, 2009, pp. 1808-1812. doi: $10.2514 / 1.36847$

[4] Raghavan, B., and Patil, M. J., "Flight Control for Flexible, HighAspect-Ratio Flying Wings," Journal of Guidance, Control, and Dynamics, Vol. 33, No. 1, 2010, pp. 64-74. doi: $10.2514 / 1.45471$

[5] Noll, T. E., Brown, J. M., Perez-Davis, M. E., Ishmael, S. D., Tiffany, G. C., and Gaier, M., "Investigation of the Helios Prototype Aircraft Mishap. Vol. 1: Mishap Report," NASA, Langley Research Center, Hampton, VA, Jan. 2004, https://www.nasa.gov/pdf/64317main_helios .pdf.

[6] Su, W., and Cesnik, C. E. S., "Nonlinear Aeroelasticity of a Very Flexible Blended-Wing-Body Aircraft," Journal of Aircraft, Vol. 47, No. 5, 2010, pp. 1539-1553. doi: $10.2514 / 1.47317$

[7] Hodges, D. H., "Geometrically Exact, Intrinsic Theory for Dynamics of Curved and Twisted Anisotropic Beams," AIAA Journal, Vol. 41, No. 6, 2003, pp. 1131-1137. doi: $10.2514 / 2.2054$

[8] Su, W., and Cesnik, C. E. S., "Strain-Based Geometrically Nonlinear Beam Formulation for Modeling Very Flexible Aircraft," International Journal of Solids and Structures, Vol. 48, Nos. 16-17, 2011, pp. 2349-2360 doi:10.1016/j.ijsolstr.2011.04.012

[9] Rodden, W. P., and Johnson, E. H., MSC Nastran 2016: Aeroelastic Analysis User's Guide, MacNeal-Schwendler Corp., Santa Ana, CA, 2016.

[10] Di Vincenzo, F. G., and Castrichini, A., Hybrid Static Aeroelasticity Toolkit (HSA) User's Guide, MSC.Software Toulouse, Toulouse, France, 2013, pp. 31-54.

[11] Riso, C., Di Vincenzo, F. G., Ritter, M., Cesnik, C.E. S., and Mastroddi, F., "A FEM-Based Approach for Nonlinear Aeroelastic Trim of Highly 
Flexible Aircraft," 17th International Forum on Aeroelasticity and Structural Dynamics (IFASD), Paper IFASD-2017-170, June 2017.

[12] MSC, MSC Nastran 2016: Non-Linear User's Guide SOL400, MacNeal-Schwendler Corp., Santa Ana, CA, 2016.

[13] MSC, MSC Nastran 2016: Quick Reference Guide, MacNealSchwendler Corp., Santa Ana, CA, 2016.

[14] Ritter, M., Dillinger, J., and Meddaikar, Y. M. "Static and Dynamic Aeroelastic Validation of a Flexible Forward Swept Composite Wing," 58th AIAA/ASCE/AHS/ASC Structures, Structural Dynamics, and Materials Conference, AIAA SciTech Forum, AIAA Paper 2017-0637, Jan. 2017.

doi: $10.2514 / 6.2017-0637$

[15] Cesnik, C. E. S., Senatore, P. J., Su, W., Atkins, E. M., and Shearer, C. M., "X-HALE: A Very Flexible UAV for Nonlinear Aeroelastic Tests," AIAA Journal, Vol. 50, No. 12, 2012, pp. 2820-2833. doi:10.2514/1.J051392

[16] Jones, J. R., and Cesnik, C. E. S., "Preliminary Flight Test Correlations of the X-HALE Aeroelastic Experiment," Aeronautical Journal of The Royal Aeronautical Society, Vol. 119, No. 1217, Feb. 2015, pp. 855-870. doi:10.1017/S0001924000010952

[17] Rodden, W. P., and Love, J. R., "Equations of Motion of a Quasisteady Flight Vehicle Utilizing Restrained Static Aeroelastic Characteristics," Journal of Aircraft, Vol. 22, No. 9, 1985, pp. 802-809. doi: $10.2514 / 3.45205$

[18] MSC, MSC Nastran 2016: Linear Static Analysis User's Guide, MacNeal-Schwendler Corp., Santa Ana, CA, 2016.
[19] MSC, MSC Nastran 2016: Dynamic Analysis User's Guide, MacNealSchwendler Corp., Santa Ana, CA, 2016.

[20] Di Vincenzo, F. G., Linari, M., Mohdzawawi, F., and Morlier, J., "Nonlinear Aeroelastic Steady Simulation Applied to Highly Flexible Blades for MAV," 17th International Forum on Aeroelasticity and Structural Dynamics (IFASD), Paper IFASD2017-056, June 2017.

[21] Neto, A. B. G., Silva, R. G. A., Paglione, P., and Silvestre, F. J., "Formulation of the Flight Dynamics of Flexible Aircraft Using General Body Axes," AIAA Journal, Vol. 54, No. 11, 2016, pp. 3516-3534. doi:10.2514/1.J054752

[22] Teixeira, P. C., and Cesnik, C. E. S., "Inclusion of Propeller Effects on Aeroelastic Behavior of Very Flexible Aircraft," 17th International Forum on Aeroelasticity and Structural Dynamics (IFASD), Paper IFASD-2017-194, June 2017.

[23] Ritter, M., Cesnik, C. E. S., and Krüger, W. R., "An Enhanced Modal Approach for Large Deformation Modeling of Wing-Like Structures," AIAA SciTech Forum, 56th AIAA/ASCE/AHS/ASC Structures, Structural Dynamics, and Materials Conference, AIAA Paper 20150176, Jan. 2015 doi: $10.2514 / 6.2015-0176$

[24] Ritter, M., Jones, J. R., and Cesnik, C. E. S., "Enhanced Modal Approach for Free-Flight Nonlinear Aeroelastic Simulation of Very Flexible Aircraft," AIAA SciTech Forum, 15th Dynamics Specialists Conference, AIAA Paper 2016-1794, Jan. 2016. doi: $10.2514 / 6.2016-1794$ 